\title{
Absolutely continuous random power series in reciprocals of Pisot numbers
}

\author{
J. Neunhäuserer \\ University of Applied Science Berlin \\ neunchen@aol.com
}

\begin{abstract}
We construct a continuum of shift invariant Borel probability measures on $\{-1,1\}^{\mathbb{N}}$ such that corresponding random power series in reciprocals of Pisot numbers are absolutely continuous with respect to the Lebsgue measure.
\end{abstract}

MSC2010: 26A46, 26A30, 28A80

Key-words: Random powers series, absolute continuity, Pisot numbers

\section{Introduction}

Let $\beta \in(0.5,1)$ be the reciprocal of a Pisot number $\alpha \in(1,2)$; this is an algebraic integer with all conjugates inside the unite circle, see Bertin et al. (1992). Let $\mathfrak{M}$ be the compact and convex space of all shift invariant Borel probability measures on $\{-1,1\}^{\mathbb{N}}$ with the weak*-topology, see Walters (1982). $\mu \in \mathfrak{M}$ induces a random power series by

$$
X_{\beta}=\sum_{i=1}^{\infty} X_{i} \beta^{i}
$$

where $\left(X_{i}\right)$ is the stationary process taking values in $\{-1,1\}$ according to $\mu$. Let $\mu_{\beta}$ be the Borel probability measure on the real line describing the distribution of $X_{\beta} ; \mu_{\beta}(B)=$ $P\left(X_{\beta} \in B\right)$. It is a classical result that goes back to Paul Erdös, that $\mu_{\beta}$ is singular if $\left(X_{i}\right)$ is a Bernoulli process. In fact Erdös (1939) proved the result for the equal weighted Bernoulli measure. The proof can easily be generalized to arbitrary homogenous, see Lalley (1999), and even inhomogeneous Bernoulli processes, see Bisbas and Neunhäuserer (2011). We are interested here in measures $\mu \in \mathfrak{M}$ such that $\mu_{\beta}$ is absolutely continuous with respect to the Lebesgue measure. We consider the set

$$
\mathfrak{A}_{\beta}=\left\{\mu \in \mathfrak{M} \mid \mu_{\beta} \text { is absolutly contiunues }\right\}
$$

and prove here the following:

Theorem $1.1 \mathfrak{A}_{\beta}$ is a continuum; a compact and convex space with more than one element. 
It is easy to infer from known results that $\mathfrak{A}_{\beta}$ contains the Parry measure $\mu$ with measure theoretic entropy $H(\mu)=\log \left(\beta^{-1}\right)$. We give detailed references in the proof of proposition 2.1 below. Furthermore using the Garsia entropy we obtain, that the space $\mathfrak{A}_{\beta}$ is compact and convex, see corollary 2.1 below. The main aim of this paper is to construct a measure $\mu \in \mathfrak{A}_{\beta}$ with measure theoretic entropy $H(\mu)>\log \left(\beta^{-1}\right)$. This proves the theorem, see section 3 .

\section{Garsia entropy}

We define a coding map $\pi:\{-1,1\}^{\mathbb{N}} \longmapsto \mathbb{R}$ by

$$
\pi\left(\left(s_{i}\right)\right)=\sum_{i=1}^{\infty} s_{i} \beta^{i} .
$$

$\mu \in \mathfrak{M}$ induces a Borel probability measure on $\mathbb{R}$ by $\mu_{\beta}=\pi(\mu)=\mu \circ \pi^{-1}$, which obviously is the distribution of the random power series $X_{\beta}$ given in the introduction.

Let $\mathcal{C}_{n}$ be the partition of $\{-1,1\}^{\mathbb{N}}$ into cylinder sets of length $n \geq 1$. The Shannon entropy of a partition with respect to $\mu$ is given by

$$
H_{\mu}\left(\mathcal{C}_{n}\right)=-\sum_{C \in \mathcal{C}_{n}} \mu(C) \log (\mu(C))
$$

As a convention we use logarithm to base 2 throughout the paper. Since $\mathcal{C}_{n+m}=\mathcal{C}_{n} \vee$ $\sigma^{-n} \mathcal{C}_{m}$ for the shift map $\sigma$ and the join of partitions $\vee$, it is easy to check that $H_{\mu}\left(\mathcal{C}_{n}\right)$ is a subadditive sequence for shift invariant measures $\mu \in \mathfrak{M}$. Hence the measure theoretic entropy of $\mu$

$$
H(\mu)=\lim _{n \longmapsto \infty} \frac{H_{\mu}\left(\mathcal{C}_{n}\right)}{n}
$$

exists. Now we define a sequence of relations $\sim_{n}$ on $\{-1,1\}^{\mathbb{N}}$ by

$$
\left(s_{i}\right) \sim_{n}\left(t_{i}\right): \Leftrightarrow \sum_{i=1}^{n} s_{i} \beta^{i}=\sum_{i=1}^{n} t_{i} \beta^{i} .
$$

Obviously this are equivalence relations. Let $\mathcal{P}_{n}$ be the partition of $\{-1,1\}^{\mathbb{N}}$ induced by $\sim_{n}$. Observe that the partition $\mathcal{P}_{n} \vee \sigma^{-n} \mathcal{P}_{m}$ is finer than $\mathcal{P}_{n+m}$. Hence by well known properties of the partition entropy, $H_{\mu}\left(\mathcal{P}_{n}\right)$ is a subadditive sequence, see Walters (1982). Hence the Garsia entropy

$$
G_{\beta}(\mu)=\lim _{n \longmapsto \infty} \frac{H_{\mu}\left(\mathcal{P}_{n}\right)}{n}
$$

exists; compare with the papers of Garsia (1962) and Garsia (1963). Note that the partition $\mathcal{C}_{n}$ is finer than the partition $\mathcal{P}_{n}$. Hence $G(\mu) \leq H(\mu)$. To be more precise we have

$$
H(\mu)=G_{\beta}(\mu)+\lim _{n \longmapsto \infty} \frac{H_{\mu}\left(\mathcal{C}_{n} \mid \mathcal{P}_{n}\right)}{n},
$$


where $H_{\mu}\left(\mathcal{C}_{n} \mid \mathcal{P}_{n}\right)$ is the conditional entropy, see Walters (1982) for the definition. The first result on the Garsia entropy, we will use, is:

Proposition 2.1 The map $\mu \longmapsto G_{\beta}(\mu)$ is upper semicontinuous and affine with maximum $\log \left(\beta^{-1}\right)$ on $\mathfrak{M}$.

Proof. Upper semicontinuity and affinity follow from proposition 6.4 of Neunhäuserer (2007), where we proved the result in a more general setting. Furthermore we know that the number of elements in the partition $\mathcal{P}_{n}$ is bounded by $\sharp \mathcal{P}_{n} \leq C \beta^{-n}$ for a constant $C>0$ depending only on $\beta$, see Garsia (1962) or Lalley (1999). Hence

$$
H_{\mu}\left(\mathcal{P}_{n}\right) \leq \log \left(\sharp \mathcal{P}_{n}\right) \leq \log (C)+n \log \left(\beta^{-1}\right),
$$

which implies $G_{\beta}(\mu) \leq \log \left(\beta^{-1}\right)$. Now consider the $\beta$-subshift $X_{\beta} \subseteq\{-1,1\}^{\mathbb{N}}$ with topological entropy $\log \left(\beta^{-1}\right)$, see definition 3.1 and corollary 3.6 of Ito and Takahashi (1974). If $\beta^{-1} \in(1,2)$ is a Pisot number this subshift is known to be Markovian by theorem 2 of Ito and Takahashi (1974). Let $\mu$ be the Parry measure on $X_{\beta}$, this is the measure of full entropy $H(\mu)=\log \left(\beta^{-1}\right)$, see Parry (1964). It is known that $\pi: X_{\beta} \longmapsto \mathbb{R}$ is invertible up to a countable set of sequences, see proposition 3.2 of Ito and Takahashi (1974). Hence there are no relations $\sim_{n}$ on $X_{\beta}$ and we have $G_{\beta}(\mu)=H(\mu)=\log \left(\beta^{-1}\right)$ for the Parry measure $\mu$.

Now we state a characterization of absolutely continuous measures $\mu_{\beta}$ using the Garsia entropy, which is implicitly contained in the work of Lalley (1999).

Proposition 2.2 For $\mu \in \mathfrak{M}$ the measure $\mu_{\beta}$ is absolutely continuous if and only if $G_{\beta}(\mu)=\log \left(\beta^{-1}\right)$.

Proof. From proposition 3 of Lalley (1999) we know that

$$
\operatorname{dim}_{H} \mu_{\beta} \leq-G_{\beta}(\mu) / \log \beta
$$

for the Hausdorff dimension $\operatorname{dim}_{H}$ of the measure $\mu_{\beta}$. If $\mu_{\beta}$ is absolutely continuous, we have $\operatorname{dim}_{H} \mu_{\beta}=1$ and hence $G_{\beta}(\mu)=\log \beta^{-1}$. On the other hand using the proof of proposition 5 of Lalley (1999) (which essential goes back to Garsia (1962), we see that $G_{\beta}(\mu)<\log \beta^{-1}$ if $\mu_{\beta}$ is not absolutely continuous. In fact Lalley assumed that $\mu_{\beta}$ is singular in the proposition, but the proof works if we have a singular part in the Lebesgue decomposition of $\mu_{\beta}$, compare with the proof of theorem 9.1 of Neunhäuserer (2007).

Since $\mathfrak{M}$ is compact and convex the last two propositions have the following consequence:

Corollary $2.1 \mathfrak{A}_{\beta}$ is nonempty, compact and convex. 


\section{Construction of a measure with full Garsia entropy}

For $i \geq 1$ we define a Borel probability measure $\nu_{i}$ on $\{-1,1\}^{\mathbb{N}}$ by

$$
\nu_{i}(C)=\frac{1}{2^{j-i \sharp} \mathcal{P}_{i} \sharp_{i} C}
$$

for cylinder sets $C \in \mathcal{C}_{j}$ with $j \geq i$. Here $\sharp$ denotes the cardinality of a set and $\sharp_{i} C:=$ $\sharp\left\{D \in \mathcal{C}_{i} \mid D \subseteq P, C \subseteq P \in \mathcal{P}_{i}\right\}$. Note that

$$
\sum_{C \in \mathcal{C}_{j}} \nu_{i}(C)=\sum_{C \in \mathcal{C}_{i}} \frac{1}{\sharp \mathcal{P}_{i} \sharp_{i} C}=\sum_{P \in \mathcal{P}_{i}} \frac{1}{\sharp \mathcal{P}_{i}}=1
$$

for any $i \geq 1$. The cylinder sets of length $j \geq i$ form a basis of the weak* topology on the space of probability measures on $\{-1,1\}^{\mathbb{N}}$, thus the measures $\nu_{i}$ are well defined. Our problem is, that the measures $\nu_{i}$ are not shift invariant. Instead of $\nu_{i}$ we will use a weak* accumulation point $\mu$ of the sequence

$$
\mu_{k}=\frac{1}{k} \sum_{i=0}^{k-1} \nu_{i} \circ \sigma^{-k}
$$

By construction $\mu$ is shift invariant, $\mu \in \mathfrak{M}$, and we will show:

Proposition 3.1 For the measure constructed above we have

$$
G_{\beta}(\mu)=\log \left(\beta^{-1}\right)
$$

The proof is based on the following lemma.

Lemma 3.1 For all $i>0$ and $0<q<i$ we have

$$
\frac{H_{\mu_{i}}\left(\mathcal{P}_{i}\right)}{i} \leq \frac{H_{\mu_{i}}\left(\mathcal{P}_{q}\right)}{q}+\frac{2 \log (2) q}{i} .
$$

The same is true for the partitions $\mathcal{C}_{i}$.

Proof. We prove the lemma for the partitions $\mathcal{P}_{i}$. The proof for $\mathcal{C}_{i}$ is the same. In the proof we write $\mathcal{P}_{1} \preceq \mathcal{P}_{2}$, if the partition $\mathcal{P}_{2}$ is a refinement of the partition $\mathcal{P}_{1}$. $\mathcal{P}_{1} \vee \mathcal{P}_{2}$ denotes the join of the partitions as usual.

By the definition of the partitions $\mathcal{P}_{i}$ we have

$$
\mathcal{P}_{i+j} \preceq \mathcal{P}_{i} \vee \sigma^{-i}\left(\mathcal{P}_{j}\right)
$$

for all $i, j \geq 1$. Hence for all $a, q \geq 1$ we get

$$
\mathcal{P}_{a q} \preceq \bigvee_{u=0}^{a-1} \sigma^{-u q}\left(\mathcal{P}_{q}\right)
$$


Given $i>0,0<q<i$ and $0 \leq k<q$ we write

$$
i=a(k) q+r+k
$$

where $a(k)=\lfloor(i-k) / q\rfloor$ and $0 \leq r<q$. Using this decomposition we have

$$
\mathcal{P}_{i} \preceq \bigvee_{u=0}^{a(k)-1} \sigma^{-u q+k}\left(\mathcal{P}_{q}\right) \vee \sigma^{-(a(k) q+k)}\left(\mathcal{P}_{r}\right) \vee \mathcal{P}_{k}
$$

By well known properties of the partition entropy $H$, see again Walters (1982), we conclude

$$
\begin{gathered}
H_{\nu_{i}}\left(\mathcal{P}_{i}\right) \leq \sum_{u=0}^{a(k)-1} H_{\nu_{i}}\left(\sigma^{-u q+k}\left(\mathcal{P}_{q}\right)\right)+H_{\nu_{i}}\left(\sigma^{-(a(k) q+k)}\left(\mathcal{P}_{r}\right)\right)+H_{\nu_{n}}\left(\mathcal{P}_{k}\right) \\
\leq \sum_{u=0}^{a(k)-1} H_{\nu_{n}}\left(\sigma^{-u q+k}\left(\mathcal{P}_{q}\right)\right)+2 q \log 2 .
\end{gathered}
$$

The last inequality follows from the fact, that the partitions $\mathcal{P}_{q}$ and $\mathcal{P}_{r}$ have less than $2^{q}$ elements. Now summing over $k$ gives

$$
q H_{\nu_{i}}\left(\mathcal{P}_{i}\right) \leq \sum_{k=0}^{q-1} \sum_{u=0}^{a(k)-1} H_{\nu_{n}}\left(\sigma^{-u q+k}\left(\mathcal{P}_{q}\right)\right)+2 q^{2} \log 2 \leq i H_{\mu_{i}}\left(\mathcal{P}_{q}\right)+2 q^{2} \log 2 .
$$

Dividing by $i q$ gives the result.

Proof of proposition 3.1. By definition of $\nu_{i}$ we have

$$
H_{\nu_{i}}\left(\mathcal{P}_{i}\right)=\sum_{P \in \mathcal{P}_{i}} \frac{1}{\sharp \mathcal{P}_{i}} \log \left(\sharp \mathcal{P}_{i}\right)=\log \left(\sharp \mathcal{P}_{i}\right) .
$$

By $\sharp \mathcal{P}_{i}>c \beta^{-i}$ and lemma 2.1 we get

$$
\log \beta^{-1}+\frac{c}{i} \leq \frac{H_{\mu_{i}}\left(\mathcal{P}_{q}\right)}{q}+\frac{2 \log (2) q}{i} .
$$

Considering the limit $i \longmapsto \infty$ and using upper semicontinuity of $H$. $\left(\mathcal{P}_{q}\right)$, we have

$$
\log \left(\beta^{-1}\right) \leq \frac{H_{\mu}\left(\mathcal{P}_{q}\right)}{q}
$$

for all $q \geq 1$. Using the subadditivity of $H_{\mu}\left(\mathcal{P}_{q}\right)$ we obtain $\log \left(\beta^{-1}\right) \leq G_{\beta}(\mu)$. On the other hand we have $G_{\beta}(\mu) \leq \log \left(\beta^{-1}\right)$ by proposition 2.1 .

From proposition 2.2 we conclude that $\mu_{\beta}$ is absolutely continuous, $\mu \in \mathfrak{A}_{\beta}$. To get theorem 1.1 it remains to show that $\mu$ is not the Parry measure with entropy $\log \left(\beta^{-1}\right)$. This follows from: 
Proposition 3.2 For the measure constructed above we have

$$
H(\mu) \geq \log \left(\beta^{-1}\right)+\liminf _{i \longmapsto \infty} \frac{1}{\sharp \mathcal{P}_{i}} \sum_{P \in \mathcal{P}_{i}} \frac{\log (\sharp P)}{i}>\log \left(\beta^{-1}\right) .
$$

Proof. By definition of $\nu_{i}$ we have

$$
H_{\nu_{i}}\left(\mathcal{C}_{i}\right)=\sum_{C \in \mathcal{C}_{i}} \frac{1}{\sharp \mathcal{P}_{i} \sharp_{i} C} \log \left(\sharp \mathcal{P}_{i} \sharp_{i} C\right)=\sum_{P \in \mathcal{P}_{i}} \frac{1}{\sharp \mathcal{P}_{i}} \log \left(\sharp \mathcal{P}_{i} \sharp P\right)=\log \left(\sharp \mathcal{P}_{i}\right)+\frac{1}{\sharp \mathcal{P}_{i}} \sum_{P \in \mathcal{P}_{i}} \log (\sharp P)
$$

and by $\sharp \mathcal{P}_{i}>c \beta^{-i}$ and lemma 2.1 for the partitions $\mathcal{C}_{i}$ we get

$$
\log \left(\beta^{-1}\right)+\frac{c}{i}+\frac{1}{\sharp \mathcal{P}_{i}} \sum_{P \in \mathcal{P}_{i}} \frac{\log (\sharp P)}{i} \leq \frac{H_{\mu_{i}}\left(\mathcal{C}_{q}\right)}{q}+\frac{2 \log (2) q}{i} .
$$

Considering the limit $i \longmapsto \infty$ and using upper semicontinuity of $H .\left(\mathcal{P}_{q}\right)$ we have

$$
\log \left(\beta^{-1}\right)+\liminf _{i \longmapsto \infty} \frac{1}{\sharp \mathcal{P}_{i}} \sum_{P \in \mathcal{P}_{i}} \frac{\log (\sharp P)}{i} \leq \frac{H_{\mu}\left(\mathcal{C}_{q}\right)}{q}
$$

for all $q \geq 1$. It remains to show that the limes inferior here is not zero. To this end consider

$$
\mathcal{P}_{i, \rho}=\left\{P \in \mathcal{P}_{i} \mid \frac{\log (\sharp P)}{i} \geq \rho\right\}
$$

for $\rho>0$ and the projection $\tilde{\mathcal{P}}_{i, \rho}=\pi\left(\mathcal{P}_{i, \rho}\right)$ to the real line. In the following let $\ell$ be the Lebesgue measure on $\mathbb{R}$. It follows from Theorem 1.1 of Feng and Sidorov (2011) using Egorov's Theorem that there are constants $\rho, \delta>0$ such that $\ell\left(\bigcup \tilde{\mathcal{P}}_{i, \rho}\right)>\delta$ for all $i$ that are sufficient large. Note that $\ell(\pi(P))=2 \beta^{i+1} /(1-\beta)$ for all $P \in \mathcal{P}_{i}$. Hence we obtain

$$
\sharp \mathcal{P}_{i, \rho} \geq \frac{\delta}{2}(1-\beta) \beta^{-i-1}
$$

for sufficient large $i$. Now we get

$$
\liminf _{i \longmapsto \infty} \frac{1}{\sharp \mathcal{P}_{i}} \sum_{P \in \mathcal{P}_{i}} \frac{\log (\sharp P)}{i} \geq \liminf _{i \longmapsto \infty} \frac{1}{\sharp \mathcal{P}_{i}} \sum_{P \in \mathcal{P}_{i, \rho}} \frac{\log (\sharp P)}{i} \geq \liminf _{i \longmapsto \infty} \frac{1}{\sharp \mathcal{P}_{i}} \frac{\rho \delta}{2}(1-\beta) \beta^{-i-1}>0 .
$$

For the last inequality we use the fact that $\sharp \mathcal{P}_{i} \leq C \beta^{-i}$ for some constant $C>0$ independent of $i$.

\section{References}

[1] Bertin, M.J., Decomps-Guilloux, A., Grandet-Hugot, M., Pathiaux-Delefosse, M., Schreiber, J.P., 1992. Pisot and Salem numbers. Birkhauser Verlag, Basel.

[2] Bisbas, A., Neunhäuserer, J., 2011. On inhomogeneous Bernoulli convolution and random power series. Real Analysis Exchange. vol 36. no.1, 213-222. 
[3] Erdös, P., 1939. On a family of symmetric Bernoulli convolutions. Amer. J. Math. 61, 974-975.

[4] Feng, D.J., Sidorov, N., 2011, Growth rate for beta expansions. Monatsh. Math. 162, 41-60.

[5] Garsia, A.M., 1963. Entropy and singularity of infinite convolutions. Pac. J. Math. 13, 1159-1169.

[6] Garsia, A.M., 1962. Arithmetic properties of Bernoulli convolutions. Trans. Amer. Math. Soc. 162, 409-432.

[7] Ito, S., Takahashi, Y., 1974. Markov subshifts and the relization of $\beta$-expansion, J. Math. Soc. Japan nol. 26. no. 1., 33-55.

[8] Lalley, S.P., 1999. Random series in powers of algebraic integers. J. London Math. Soc 57 no. 3, 629-654.

[9] Neunhäuserer, J., 2007. Random walks on infinite self-similar graphs. Electronic Journal of Probability. vol.12. no. 46, 1258-1275.

[10] Parry, W., 1964, Intrinsic Markov chains, Trans. Amer. Math. Soc. 112, 55-64, 1964.

[11] Walters, P., 1982, An introduction to ergodic theory. Springer Verlag, Berlin. 